\title{
Clustering Versus Evenly Distributing Energy Dissipation in Wireless Sensor Routing for Prolonging Network Lifetime*
}

\author{
Guangyan Huang ${ }^{1}$, Xiaowei $\mathrm{Li}^{1}$, and Jing $\mathrm{He}^{2}$ \\ ${ }^{1}$ Advanced Test Technology Lab., Institute of Computing Technology, \\ Chinese Academy of Sciences, Beijing, P. R.China 100080 \\ \{huanggy, lxw\} aict.ac.cn \\ ${ }^{2}$ Chinese Academy of Sciences Research Center on Data Technology and \\ Knowledge Economy, Beijing, P. R. China 100080 \\ hejingegucas.ac.cn
}

\begin{abstract}
A novel Cluster Heads $(\mathrm{CH})$ choosing algorithm based on both Minimal Spanning Tree and Maximum Energy resource on sensors, named MSTME, is provided for prolonging lifetime of wireless sensor networks. MSTME can satisfy three principles of optimal CHs: to have the most energy resource among sensors in local clusters, to group approximately the same number of closer sensors into clusters, and to distribute evenly in the networks in terms of location. Simulation shows the network lifetime in MSTME excels its counterparts in two-hop and multi-hop wireless sensor networks.
\end{abstract}

\section{Introduction}

Recent advancements in wireless communications and electronics have made the development of low-cost, low-power smart wireless sensors available [1-3]. Tens or hundreds of sensors can be deployed in observation environment to sense physical parameters that are sent to the Base Station (BS) to provide useful knowledge for people [4-6]. Because sensors have limited battery resource and wireless communications consume large number of energy, energy efficiency of the communication is most important in the wireless sensor networks [7,8].

The energy consumed by wireless communications is related with the number of transmitting data and transmitting distance [9]. Optimal routes can achieve the minimal average transmitting distance. Furthermore, with only limited computing abilities, sensors can fuse data by compressing or getting rid of redundancy to reduce the number of data and then send the user-cared knowledge to BS [9]. Thus, sensors are clustered into several clusters and some sensors are chosen as Cluster Heads $(\mathrm{CH})$ to do the fusing and middle-transferring jobs. However, different transmitting distances and different jobs of non- $\mathrm{CH}$ sensors and $\mathrm{CHs}$ bring different energy dissipation. Thus, all the sensors should work as a team and evenly distributing energy dissipation to prolong the whole network lifetime that is defined as the Time before the First sensor

\footnotetext{
This paper was supported by the National Basic Research Program of China (No.2005CB321604 and No.2004CB720103), and the National Natural Science Foundation of China (No.90207002).
} 
Node Dies (TFND). Also, the Ratio of TFND and the Time before All sensor Nodes Die (TAND), RTT, is used to evaluate the effectiveness of evenly distributing energy dissipation. Therefore, network lifetime is determined by both energy efficiency and effectiveness of evenly distributing energy dissipation.

Low Energy Adaptive Clustering Hierarchy (LEACH) [9-11] gives an elegant scheme for evenly distributing energy dissipation. Base Station Controlled Dynamic Clustering Protocol (BCDCP) [12] improves LEACH by reducing the transmitting distances and balancing the number of sensors in clusters [13]. LEACH and BCDCP show network lifetime can be improved further in four aspects: better closeness of sensors in each cluster (Closer), the same number of sensors in each cluster (Same Number), CHs with the most energy resource (Maximum Energy) and locations of CHs are distributed evenly (Even Location). LEACH and BCDCP do well in Maximum Energy and in both Closer and Same Number, respectively. This paper proposes a CH-choosing method, Minimal Spanning Tree (MST) and Maximum Energy resource on sensors determining CHs (MSTME), to do best in all the four aspects.

\section{MSTME CH Choosing Algorithm}

MSTME CH choosing algorithm is given in Table 1. The main goal is to choose expected number, $N_{C H}$, of $\mathrm{CHs}$, which satisfy all of the four optimal principles. Then, non- $\mathrm{CH}$ sensors affiliate their closest $\mathrm{CHs}$ and thus clusters are formed. MSTME is reasonable for clustering while evenly distributing energy dissipation in wireless sensor networks. Firstly, MST connects all $\mathrm{CH}$ candidates with the minimal edges. Wherever the edge is broken, the nodes in the same split sub trees are closer. Because non-candidate sensors support the nearest $\mathrm{CH}$ candidates, $\mathrm{CH}$ candidates may delegate their supporters in terms of locations. Thus, sensors in the same clusters formed by MSTME are closer to each other. Also approximately the same number of supporters in each $\mathrm{CH}$ candidate subset ensures nearly the same number of sensors closest to the $\mathrm{CH}$ in this subset. At last, any nodes in MST are high-energy sensors. Therefore, MSTME synthetically does well in the four optimal principles.

Suppose BS knows the locations of all sensors and MSTME runs on BS. Maximum Energy principle is satisfied firstly, then CHs (solid dots) formed by MSTME shown in Fig. 1 (c) and (d) satisfy Closer, Same Number and Even Location better compared with LEACH and BCDCP in Fig. 1 (a) and (b). The number of CHs is greater, the MSTME method is better. However, optimal number of $\mathrm{CHs}$ is determined by network topology. Six CHs is optimal in two-hop networks according to [10], and nine $\mathrm{CHs}$ is optimal in multi-hop networks based on simulated annealing algorithm.

To simulate the network lifetime of MSTME, we implement an energy simulator of wireless sensor networks programmed in $\mathrm{C} / \mathrm{C}++$. We use a radio power model and related parameter values in [10] and a network shown in Fig. 1 with 100 nodes randomly deployed in $100 \mathrm{~m} \times 100 \mathrm{~m}$. Initial energy resource on each node, the package size of data message and BS position are $2 \mathrm{~J}, 20 \mathrm{kbits}$ and $(25 \mathrm{~m}, 150 \mathrm{~m})$, respectively.

In two-hop networks, Fig. 2 (a) shows TFND in MSTME increases $3.2 \%$ of that in LEACH and also TAND of MSTME outperforms 3.6\% that of LEACH. RTT of $96 \%$ in MSTME is nearly the same as RTT of $96.4 \%$ in LEACH. It means that MSTME gets better trade-offs on clusters with even numbers of closer sensors and $\mathrm{CHs}$ with 
more energy resources. In multi-hop networks, Fig. 2 (b) shows TFND in MSTME increases $6.4 \%$ of that in BCDCP and TAND in MSTME increases $5.6 \%$ of that in BCDCP. RTT of $98.7 \%$ in MSTME is nearly the same as RTT of $98 \%$ in BCDCP. MSTME performs better because it reduces average energy dissipation of the networks while keeps RTT approximately the same as both LEACH and BCDCP. In addition, it seems that MSTME performs better in Fig. 2 (b) than in Fig. 2 (a), because MSTME reduces average transmitting distance of $\mathrm{CHs}$ further in multi-hop networks.

Table 1. MSTME CH Choosing Algorithm

Step 1: Sensors with more energy resource than average level are selected into $\mathrm{CH}$ candidates set, $S$. Then a MST, $T$, is used to connect all the items in $S$. Also, supporters of a $\mathrm{CH}$ candidate $x$ are those non-candidate sensors that are nearest to $x$ among all $\mathrm{CH}$ candidates. Compute the number of supporters for each $\mathrm{CH}$ candidate including candidate itself.

Step 2 (Initialization): Let the number of split edge $n S p l i t=0, T^{\prime}=T$ and $S^{\prime}=S$.

Step 3 (Loop): Find an edge to break $T$ ' into two sub MSTs of $T 1$ and $T 2$ and at the same time the nodes in $S$ ' are grouped into two subsets of $S 1$ and $S 2$ respectively with the nearest number of supporters in both subsets. Then let $n$ Split $=n S p l i t+1$.

Step 4 (Termination test): If $n$ Split $>=N_{C H}$, go to Step 5. Otherwise, go on splitting $S 1$ and $S 2$ in turn. If the number of supporters in $S 1$ (or $S 2$ ) is more than $N / N_{C H}$, then let $S^{\prime}=S 1$ and $T^{\prime}=T 1$ (or $S^{\prime}=S 2$ and $T^{\prime}=T 2$ ). Go to Step 3.

Step 5: The $\mathrm{CH}$ candidate with the most energy resource in each subset is chosen as $\mathrm{CH}$.



(a)6 $\mathrm{CHs}$ in $\mathrm{LEACH}$

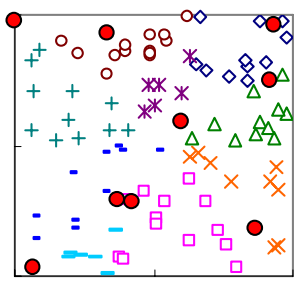

(b) 9 CHs in BCDCP



(c)6 CHs in MSTME (d)9 CHs in MSTME

Fig. 1. Analysis of $\mathrm{CH}$ Distribution

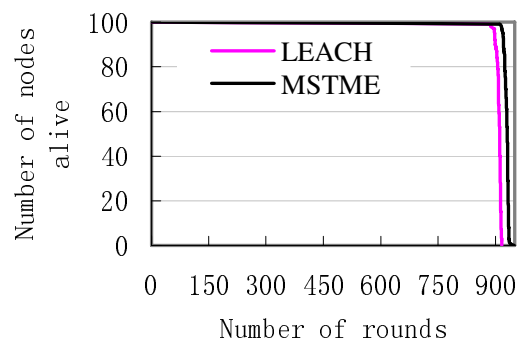

(a) Two-hop

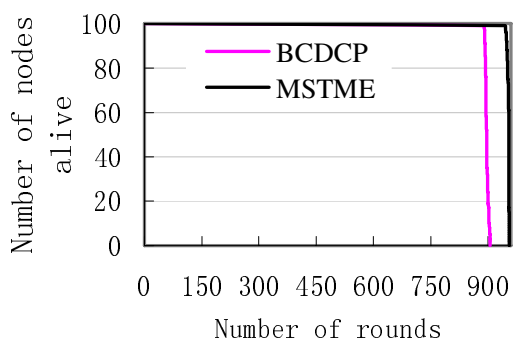

(b) Multi-hop

Fig. 2. Lifetime in Wireless Sensor Networks 


\section{Conclusions}

MSTME is proposed to cluster sensors optimally in wireless sensor networks. It not only reduces the average transmitting distance but also distributes the energy dissipation evenly in the networks. Simulating of the network lifetime shows MSTME excels LEACH and BCDCP in two-hop and multi-hop networks respectively.

\section{References}

1. Chandrakasan A., Amirtharajah R., Cho S.-H., Goodman J., Konduri G., Kulik J., Rabiner W., Wang A.: Design Considerations for Distributed Microsensor Networks. In: Proc. IEEE Custom Integrated Circuits Conference (1999) 279-286

2. Clare L., Pottie G., Agre J.: Self-Organizing Distributed Sensor Networks. In: Proc. SPIE Conf. Unattended Ground Sensor Technologies and Applications (1999) 229-237

3. Dong M., Yung K., Kaiser W.: Low Power Signal Processing Architectures for Network Microsensors. In: Proc. Int'l Symp. Low Power Electronics and Design (1997) 173-177

4. Estrin D., Govindan R., Heidemann J., Kumar S.: Next Century Challenges: Scalable Coordination in Sensor Networks. In: Proc. ACM/IEEE Mobicom (1999)

5. Kulik J., Rabiner W., Balakrishnan H.: Adaptive Protocols for Information Dissemination in Wireless Sensor Networks. In: Proc. ACM/IEEE Mobicom (1999)

6. Xu Y., Heidemann J., and Estrin D.: Geography-Informed Energy Conservation for Ad Hoc Routing. In: Proc. SIGMOBILE (2001)

7. Al-Karaki J. N., Kamal A. E.: Routing Techniques in Wireless Sensor Networks: A Survey. IEEE Wireless Communications, vol.11, (2004) 6-28

8. Akyildiz I. F., Weilian Su, Sankarasubramaniam Y., Cayirci E.: A Survey on Sensor Networks. IEEE Communications Magazine, vol. 40, (2002) 102-114

9. Heinzelman W. B., Chandrakasan A. P., Balakrishnan H.: Energy Efficient Communication Protocol for Wireless Microsensor Networks. In: Proc. Hawaii Int'l. Conf. Sys. Sci. (2000)

10. Heinzelman W. B., Chandrakasan A. P., Balakrishnan H.: An Application-Specific Protocol Architecture for Wireless Microsensor Networks. IEEE Transactions on Wireless Communications, vol.1, (2002) 660-670

11. Heinzelman W. B.: Application-Specific Protocol Architectures for Wireless Networks. Ph.D. Thesis, Massachusetts Institute of Technology (MIT), (2000)

12. Muruganathan S. D., Ma. D. C. F., Bhasin R. I., Fapojuwo A. O.: A Centralized EnergyEfficient Routing Protocol for Wireless Sensor Networks. IEEE Communications Magazine, vol.43, (2005) 8-13

13. Ghiasi S. (et al): Optimal Energy Aware Clustering in Sensor Networks. In: MDPI Sensors, vol.2, no.7 (2002) 40-50 